\title{
Class Based and Shared Resources Access Control for Distributed Video-on-Demand System
}

\author{
Sami S. Alwakeel and Muhammad Ammad-Uddin
}

\begin{abstract}
We are proposing an efficient admission control policy for Video-on-Demand (VOD) system. Traditional Admission control system uses either threshold or resource capacity to accept or reject any incoming request. The distinction of the proposed scheme from the previous work includes: (1) a new concept of integrating the both schemes (shared resource admission control and class base admission control) into one system, is introduced (2) another new concept of Probability, is introduced to access the shared resource (Ports, Bandwidth). In the proposed system, total capacity of VOD server (ports) is partitioned equal to the number of classes plus shared area. When a request arrives at VOD server, it will be admitted if there is capacity to its corresponding partition, otherwise we will check the capacity of shared area and admit it with some probability. If there is no capacity in shared area or no probability, the request will be rejected. The evaluation of the proposed system is done by the simulation model. Numbers of blocked requests are calculated for performance measure. It is observed from the simulation results that proposed admission control policy not only improves the performance of high-priority classes but also reduces a lot the total number of rejected requests.
\end{abstract}

Index Terms-Admission control, blocking probability, quality of service, video on demand, IPTV, multimedia communication.

\section{INTRODUCTION}

Video-on-Demand (VOD) system is an interactive TV technology that allows the subscribers to view programs in real time or download the programs and watch them later. A VOD system at the consumer level can consist of a standard TV receiver along with a set-top box. The services are delivered over the Internet to home computers, portable computers, high-end cellular telephone sets and advanced digital media devices.

Customer can request any video any time from anywhere. In response to a request, VOD server will deliver high quality digitized video directly to the client set-top box. A large number of video requests arrive per hour but on the other hand, VOD server has limited capacity in terms of available connection ports or bandwidth. There should be an admission control policy to decide the admission or rejection of the incoming requests to maintain quality of service at the guarantied level. Incoming request is admitted on the basis of either available resources or nature of a request. The request of a popular program should not be rejected because it can

Manuscript Received September 22, 2012; revised January 28, 2013.

The authors are with the College of Computer and Information Sciences, Computer Engineering Department, King Saud University, Saudi Arabia (e-mail: swakeel@ksu.edu.sa , amuhammad@ksu.edu.sa). generate more revenue. The proposed admission control and request handling policy utilized the resources more efficiently such that maximum of the requests should be admitted, and most of the resources are used all the time, and always have a capacity to admit the request of most popular programs. This paper is organized as follows. Related work is discussed in Section II, proposed admission control policy is illustrated in the Section III, simulation model, and results is given in Section IV, and conclusion is presented in Section V.

\section{RELATED WORK}

The author [1] describes different deterministic and predictive admission control polices. In this paper, author presented four admission control algorithms maximum, Adaptive maximum, average and average adaptive.

As describe by[2] admission control is done based on resource availability and capacity. Dynamic bandwidth sharing mechanism is used between reading and writing. In this paper threshold is not used as an admission control parameter.

[3] Says that server capacity is divided among the number of priority threshold values, based on which the system decides dynamically whether to accept clients of different priority classes. In this paper admission control is done based on hardware requirements and workload characteristics of client requests. In this paper threshold value is dynamic, depends upon the client workload characteristics.

According to the study [4] VOD architecture is divided into two categories Local and Remote, and some of the blocked requests are re-distributed to other available resources.

Traditionally, admission control methods restrict access to a resource based upon the Statistical Data [5]-[7], threshold [8] or probability [9]; This paper extends these traditional methods by introducing additional combination of class based and probabilistic restrictions in the admission control process. The purpose of enforcing probability restriction is that if the threshold level is achieved, then incoming request will not be admitted in the system directly unless some capacity is conserved for the use of more lucrative requests.

\section{PROPOSED ADMISSION CONTROL POLICY}

The proposed system Class Based and Shared Resources Access Control (CBSRAC) is different from other works it extends the threshold based admission control to probability based admission control. It is a combination of shared resource and class base admission control. The proposed 
algorithm is described as follows:

In this proposed system, VOD server capacity is partitioned as; $C 1, C 2,--C n, C s$. There are n priority classes and $\mathrm{Cs}$ is a shared Area. Request arrives with rate of $\lambda_{i}$ where $i$ is the class of Service of that request and $1<=i<=$ $\mathrm{k}$. The proposed system will admit the incoming request if there is capacity in its required class $(\mathrm{Ci}>\mathrm{Co}) \mathrm{Co}$ is occupancy of that class, If there is no capacity in the particular class $(\mathrm{Ci}=\mathrm{Co})$ then request is admitted in shared capacity class $\mathrm{Cs}$ with probability $\mathrm{Pi}$. If there is no probability / no capacity in the shared area, then call will be rejected, Number of rejected requests will be calculated for performance analysis. Input parameters for proposed algorithm are as follows:

\begin{tabular}{ll}
\multicolumn{1}{c}{ Variable } & \multicolumn{1}{c}{ Description } \\
\hline \hline$\lambda \mathrm{l}$ & Arrival request rate of class $\mathrm{i}$ \\
$\mathrm{i}$ & Classes of service $0>\mathrm{i}<\mathrm{n}$ \\
$\mathrm{K}$ & Total number of Partitions $0>\mathrm{k}<\mathrm{n}$ \\
Capacity[k] & Total ports are divide into $\mathrm{k}$ partition \\
Capacity[i] & Allocated ports for class i \\
Shared_Cap & Allocated Ports for shared area \\
shared_pob[i] & Probability of each class to use shared area \\
\hline \hline
\end{tabular}

The algorithm for proposed admission control policy is described as follow:

$/ / \lambda i$ is arrival rate of incoming request for class $\mathrm{i}$

$i=$ class of service

if (Capacity $[i]>0$ )

\{

Admitted "Admitted from reserved ports for class $i$ " Capacity $[i]$--

\}

\{

else if (Shared_Cap $>0)$

Probability $=$ Random $(1,0)$

if ( Probability $<$ shared_pob $[i]$ )

\{

Admitted "Admitted in Shared area"

Shared_Cap--

\}

\}

else \{ Rejected "No Probability"

else

\{

Rejected "No space in class $i$ and Shared Area" \}

The flow chart of algorithm for proposed admission control policy is described Fig. 1.

\section{Simulation Model}

Simulation is conducted in OMNET ++ simulation package. Simulation consists of a video server number of clients and communication link in between. Video Sever is a multithreaded server so that it can handle each request independently and each client represented each class (numbers of clients are equal to the number of class) each client generates the specific class requests at the rate of $\lambda_{1}, \lambda_{2}---\lambda_{\tilde{N}}$ The server handles each request as per allocated threshold and probability of that class.

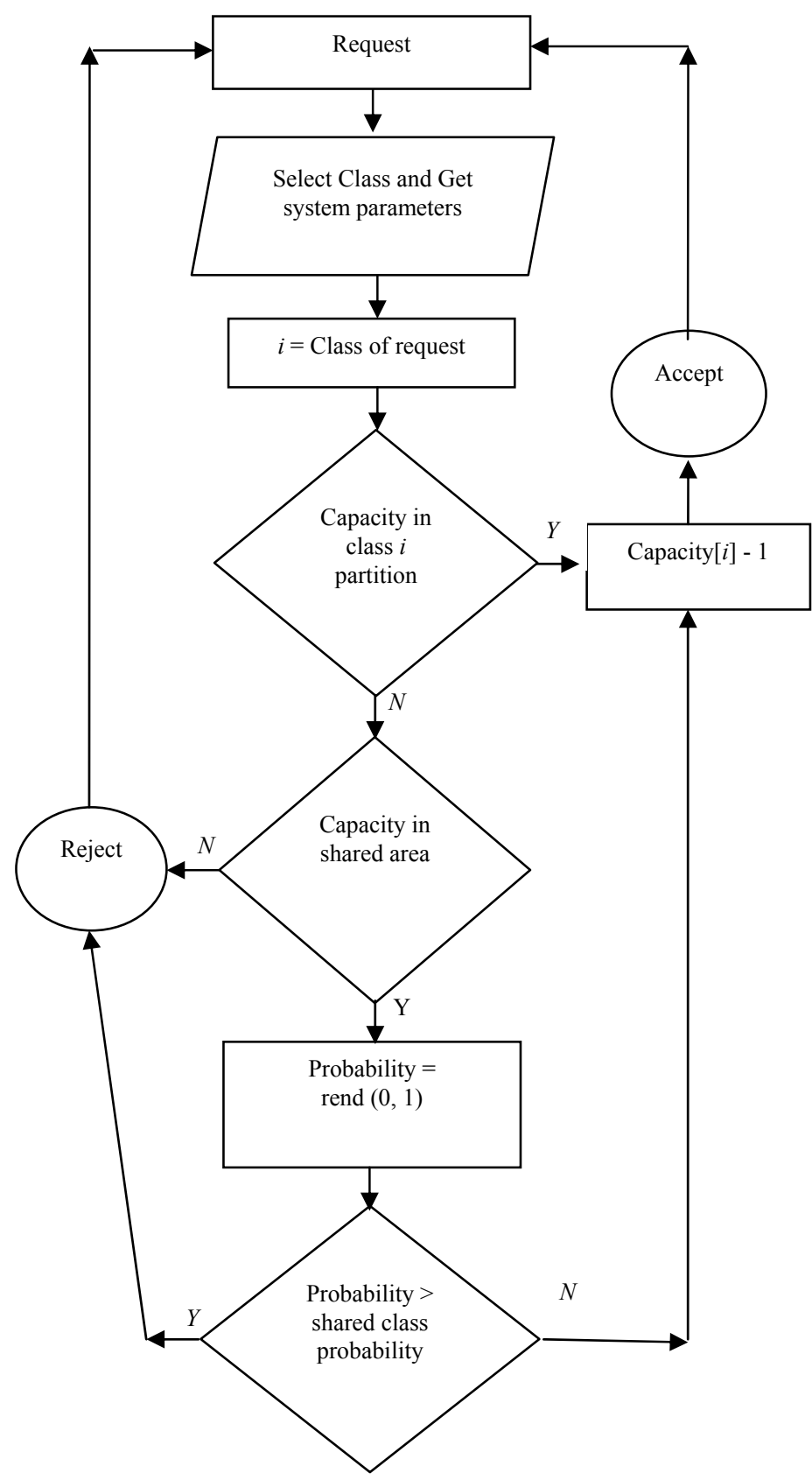

Fig. 1. BSRAC flow chart.

The proposed admission control policy is tested against varying of different parameters and conditions. Different simulation cases and simulation run are given bellow, which were conducted to test the proposed admission control policy.

\section{A. Simulation Case 1 (Varying FOr Arrival Rate of High Priority Class)}

In simulation case 1 there are two classes of service, class A popular and Class B un-popular Class A has higher priority as compare to class B. Arrival rates of incoming requests of each class is $\lambda_{1}$ and $\lambda_{2}$ respectively. Capacity of server is partitioned as $56 \%, 14 \%, 30 \%$ for class A, B and Shared Area respectively. The results are shown in Fig. 2(a) and Fig. 2(b): 

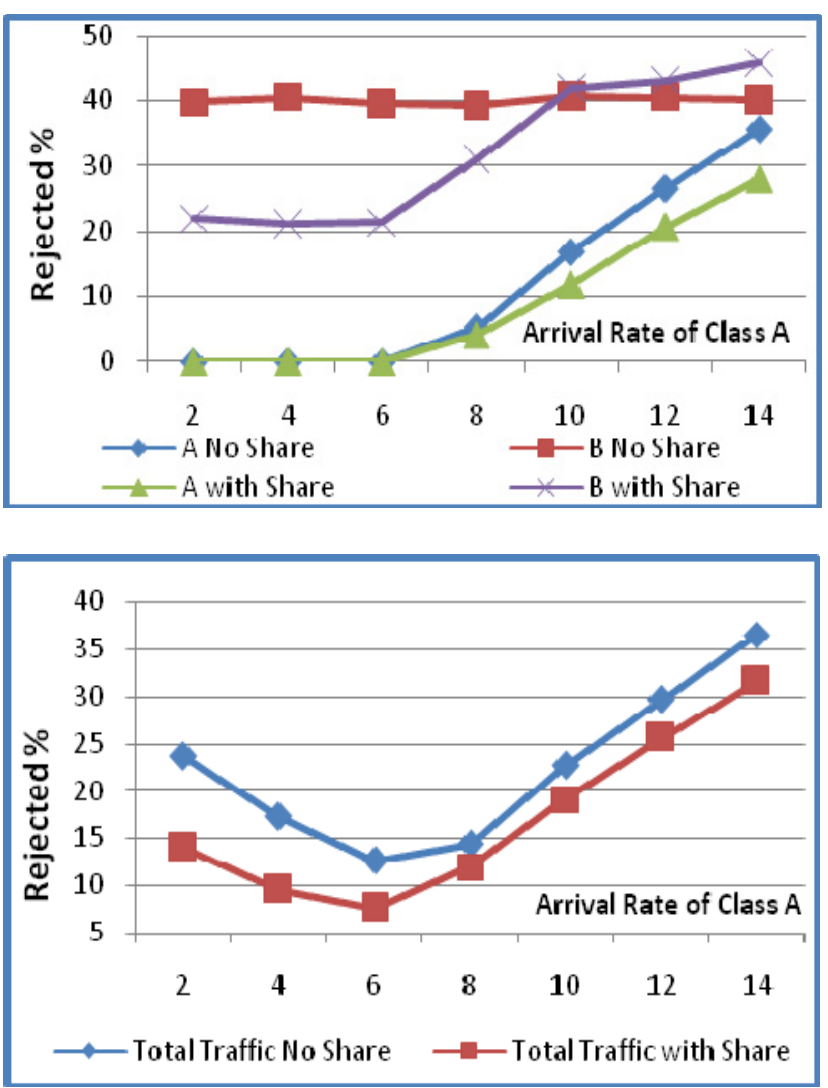

Fig. 2. (a) Rejection $\%$ of each class versus $\lambda_{1}$ (b) Rejection $\%$ of overall system versus $\lambda_{1}$

Fig. 2(a) shows the effect of varying $\lambda_{1}$ (arrival rate of popular class) where all other parameters are constant. It is very clear that the performance of high-priority class is improved a lot in proposed scheme, and even it will perform better in high arrival rate.

Fig. 2(b) Illustrate the total rejected requests with shared area and without shared area, with varying of $\lambda_{1}$. This graph shows that total rejected requests in the proposed scheme is very low as compare to the conventional scheme.

\section{B. Simulation Case 2 (Varying for Arrival Rate of Low Priority Class)}

Arrival rate of low priority class is varying and constant for high-priority class. Fig. 3(a). This graph shows that our proposed policy is also giving better results when request arrival rate of low priority class is varying. Class A and class $\mathrm{B}$ both are giving best results as compare to the conventional scheme.

Fig. 3(b). It shows the total rejected requests with shared area and without shared area with varying of $\lambda_{2}$. It describes that total rejected request are much low in our proposed system.

\section{Simulation Case 3 (Arrival Rate of High and Low Priority Class Both Are Varying)}

In simulation case 3, there are two classes of service class $\mathrm{A}$ and class $\mathrm{B}$. Class $\mathrm{A}$ has higher priority as compare class $\mathrm{B}$ and $\lambda_{1}$ and $\lambda_{2}$ both are varying ( arrival rate of both the classes is varying). Capacity of server is partitioned as $56 \%, 14 \%$, $30 \%$ for class A, B and Shared Area respectively.
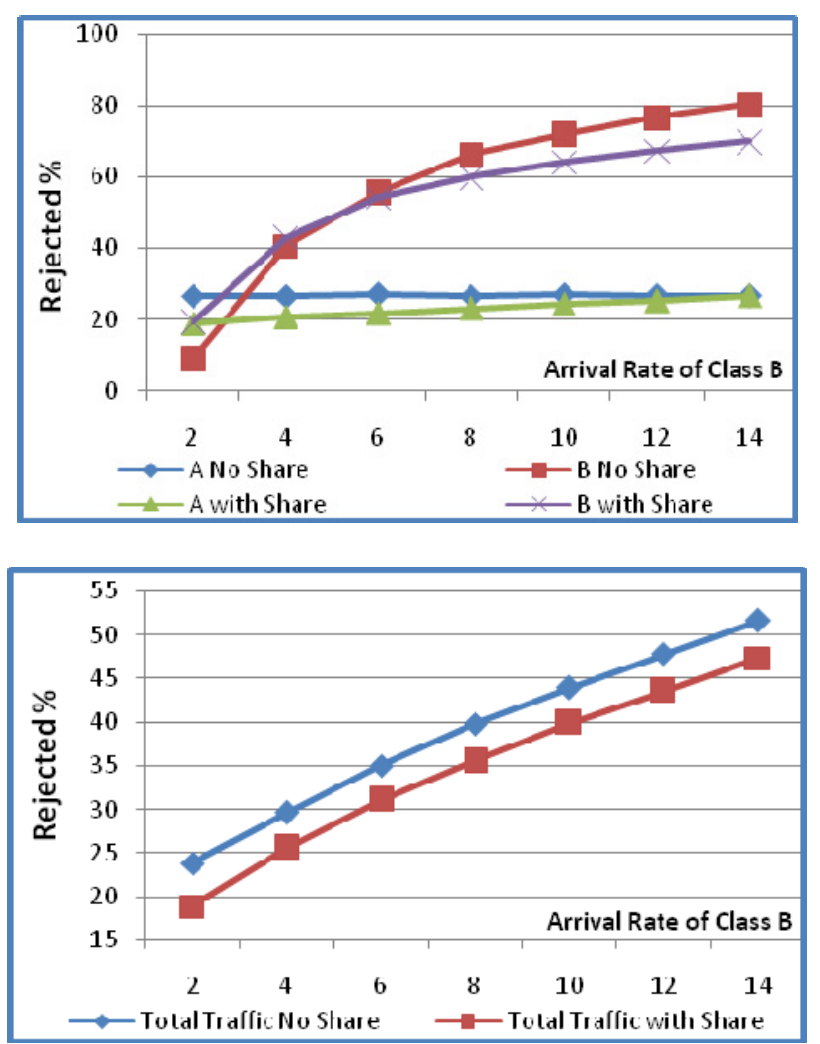

Fig. 3. (a) Rejection $\%$ of each class versus $\lambda 2$ (b) Rejection $\%$ of overall system versus $\lambda 2$
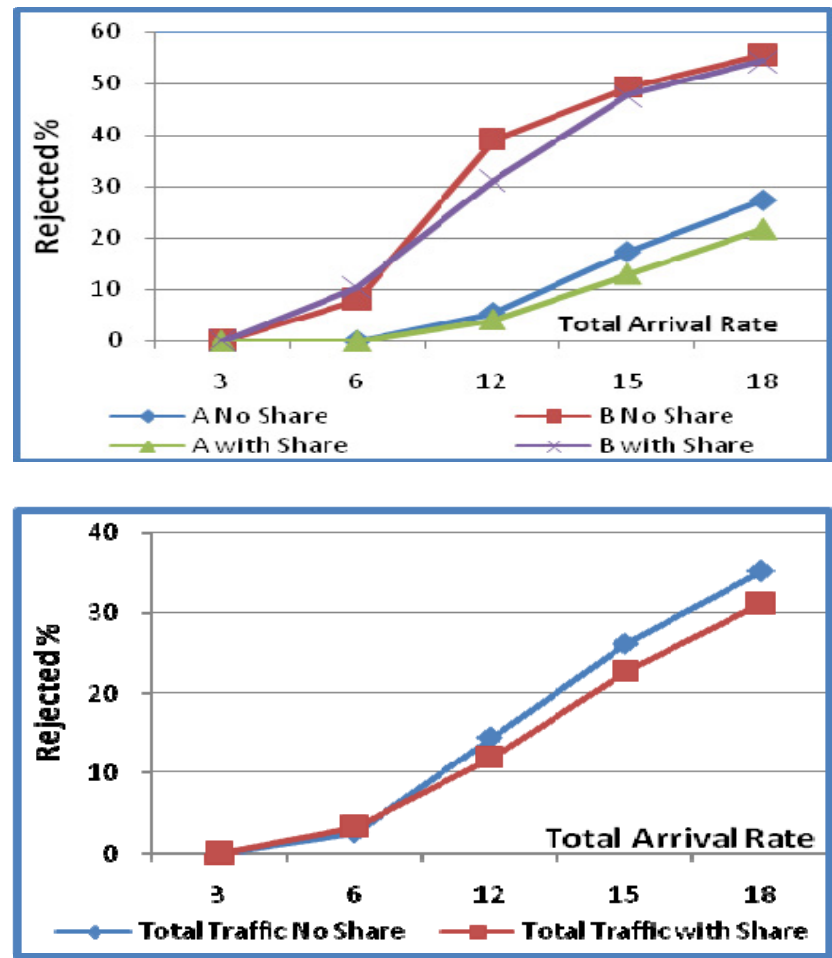

Fig. 4. (a) Rejection $\%$ of each class versus average $\lambda$ (b) Rejection $\%$ of over all system versus average $\lambda$

Fig. 4(a). It shows the rejection percentage of popular class is much improved in proposed VOD system, when arrival rates of incoming requests of both classes are varying.

The total rejected requests in the proposed system (with shared area), and conventional system (without shared area) are given in Fig. 4(b) and again proposed system has given outclass results. 


\section{Simulation Case 4 (Effect of Varying Shared Area Percentage)}

In simulation case 4, there are two classes of service class $A$ and Class B and effect of varying shared area percentage is studied, all other parameters $\lambda_{1}, \lambda_{2}$ and classes' priorities are constant, and the resultant graph is shown in Fig. 5.

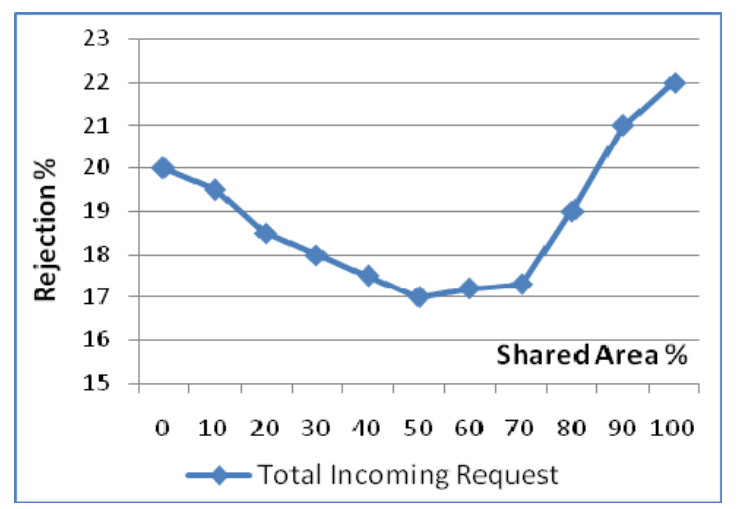

Fig. 5. Rejected requests versus shared area \%

Fig. 5 shows the effect of varying of the shared area from $0 \%$ to $100 \%$. $0 \%$ shared area means no shared area all the resources are distributed into two classes $\mathrm{A}$ and $\mathrm{B}$ proportionally, and $100 \%$ mean all the resources are put in the shared pool nothing is reserved for high-priority class A and low priority class B. It is observed in Fig. 5 that proposed policy will give best performance if shared area is $50 \%$ to $70 \%$ of total resources.

\section{E. Simulation Case 5 (four classes of services)}

The performance of proposed VOD system is also tested against four classes of services (class A, Class B, Class C and Class D). Arrival rates of the incoming requests for all the classes are same $\left(\lambda_{1}=\lambda_{2}=\lambda_{3}=\lambda_{4}\right)$ but varying for each simulations run. And server capacity is partitioned as 30,15 , 10,5 , and 40 for class A, B, C, D and shared area respectively. The performance of the proposed system is also checked against varying of different Probabilities and distinct parameters, and it is found that proposed policy performed well in all cases. One of the results is given bellow:

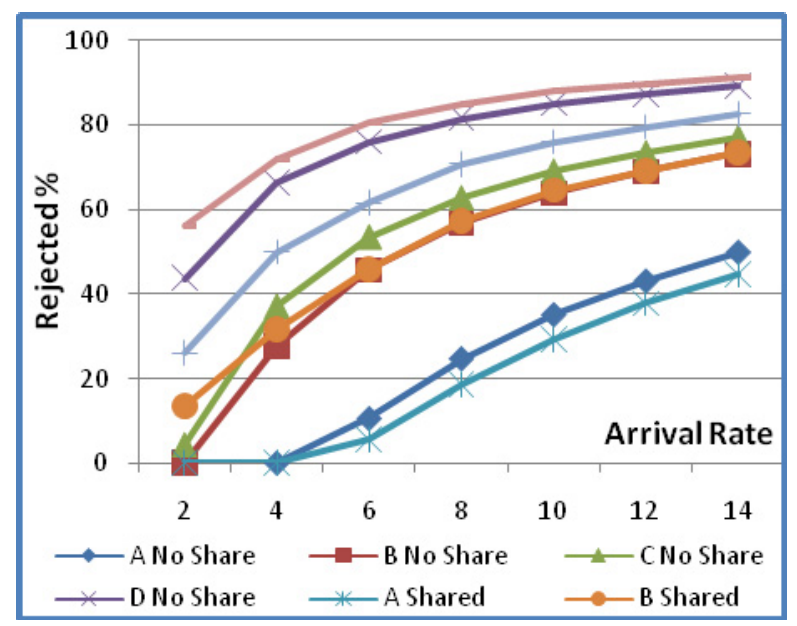

Fig. 6. Rejected requests versus arrival rate

It can be concluded from Fig. 6 that if we have many classes of service, then every high-priority class will perform better than Low priority class.

\section{CONCLUSION}

In this paper, we analyzed the performance if VOD system by comparing conventional admission control policy and proposed (CBSR) class base, shared area and probability base admission control policy. By using the simulation model, we proved that proposed admission control policy will improve the performance of high-priority class and overall performance of the system. It is also concluded that beast results can only be achieved if shared reserve is 50 to $70 \%$ of the total resources.

\section{REFERENCES}

[1] B. Qazzaz, J. Moreno, J. Xiao, P. Hernandez, R. Suppi, and E. Luque, "Admission control policies for video on demand brokers," in 2003 International Conference on Multimedia and Expo, ICME '03, 2003, pp. 529-32.

[2] R. Zimmermann and K. Fu, "Comprehensive statistical admission control for streaming media servers," in Proceedings of the eleventh ACM International Conference on Multimedia - MULTIMEDIA'03, 2003, pp. 75.

[3] I. Chen and C.-M. Chen, "Threshold-Based Admission Control Policies for Multimedia Servers,” The Computer Journal, vol. 39, no. 9, pp. 757-766, 1996.

[4] P. Mundur, R. Simon, and A. K. Sood, "End-to-End Analysis of Distributed Video-on-Demand Systems," IEEE Transactions on Multimedia, vol. 6, no. 1, pp. 129-141, Feb. 2004

[5] H. M. Vin, P. Goyal, A. Goyal, A. Goyal, and T. Hall, "A Statistical Admission Control Algorithm for Multimedia Servers," in Proceedings of the Second ACM International Conference on Multimedia, 1994, no. 512, pp. 33-40.

[6] E. Biersack and F. Thiesse, "Statistical admission control in video servers with variable bit rate streams and constant time length retrieval," in 22nd EUROMICRO Conference on Hardware and Software Design Strategies, 1996, pp. 633-639.

[7] I. Kim, J. Kim, S. Lee, and K. Chung, "Measurement-Based Adaptive Statistical Admission Control Scheme for Video-on-Demand Servers," in 15th International Conference on Information Networking, 2001, pp. 471-478.

[8] P. Mundur, A. K. Sood, and R. Simon, "Class-based access control for distributed video-on-demand systems," IEEE Transactions on Circuits and Systems for Video Technology, vol. 15, no. 7, pp. 844-853, Jul. 2005.

[9] F. Y. S. Lin, "Optimal real-time admission control algorithms for the video-on-demand (VOD) service," IEEE Transactions on Broadcasting, vol. 44, no. 4, pp. 402-408, 2010.

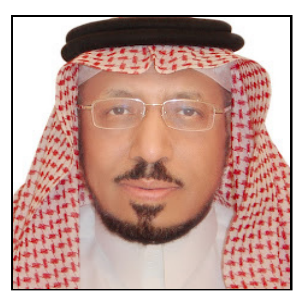

Sami S. Alwakeel is a professor of Department of Computer Engineering, CCIS, KSU. He received his B.Sc. Degree in electrical engineering with honor degree 1st class from King Saud University, Riyadh Saudi Arabia in June 1975 and M.Sc. Degrees from Department of Electrical Engineering, Stanford University, California. U.S.A. in 1978, and also Ph.D. Ddegrees from Department of Electrical Engineering, Stanford University, Calif., U.S.A. in 1983.

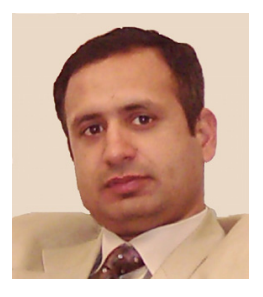

Muhammad Ammad-Uddin is a researcher of Department of Computer Engineering, CCIS, KSU. He obtained his M.S Computer Networks (MS) Degree in Wireless Networks from COMSATS Institute of Information Technology Islamabad Pakistan 2004 -2006. And he obtained his Master of Computer Sciences (MCS) Degree in Software Engineering from Bahria University, Islamabad, Pakistan from 1999 to 2001. 